\title{
MedienPädagogik
}

Zeitschrift für Theorie und Praxis der Medienbildung

\section{Autorität von Medientechnik - Effekte sozio-materieller Arrangements in der Schule}

\author{
Katharina Bock
}

\begin{abstract}
Zusammenfassung
Dieser Beitrag rückt das Zusammenspiel von menschlichen Aktivitäten und materiellen Gegebenheiten sowie mögliche, damit einhergehende hierarchisch-machtvolle Effekte in den Mittelpunkt - eine Perspektive, die in der erziehungswissenschaftlichen Bildungsforschung bisher noch wenig Beachtung findet. Hierbei wird der Materialisierungsprozess solch machtvoller Verhältnisse nachgezeichnet und aufgezeigt, auf welchem Weg technische Geräte und Infrastrukturen Autorität i.S.v. Macht erlangen und so in die Lage versetzt werden, das Handeln insbesondere von Schüler/innen zu determinieren. Dieser Materialisierungsprozess wird an der untersuchten Institution, einer Integrierten Gesamtschule, massgeblich unterstützt durch einen `Bring Your Own Device`(BYOD)-Ansatz, der darauf basiert, die Ausstattung von Kindern und Jugendlichen mit privaten, mobilen Geräten im schulischen Kontext zu nutzen. Der Beitrag präsentiert einen ethnografischen Bericht, der herausarbeitet, welche Bedeutung die Schule bzw. deren (didaktische) Leitung medienbezogenen Lehr-/Lernprozessen und dem BYOD-Ansatz zuschreibt, welche konzeptuellen und technischen Voraussetzungen daran geknüpft und inwieweit diese gegeben sind. Zudem wird herausgearbeitet, wie der BYOD-Ansatz realisiert wird und welche Effekte die Art der Umsetzung insbesondere für die sbetroffenen schüler/innen hat.
\end{abstract}

Authority of Media Technology - Effects of Socio-Material Arrangements in School

\begin{abstract}
This article takes a perspective that has so far received little attention in educational research: the interwovenness of material entities with human bodies, and possible, associated hierarchical-powerful effects. The article traces the materialization process of powerful conditions and shows how technical artefacts and infrastructures acquire (pedagogical) authority, which enables media technology to affect the conditions in the classroom. At the studied institution, a comprehensive school, this materialization process is significantly supported by a >Bring Your Own Device (BYOD) approach. The approach is based on using student's private, mobile devices at school. The paper presents an ethnographic report that examines what importance is attributed to media-related teaching/learning processes and the BYOD approach by the studied school, which conceptual and technical requirements are attached to them and to what extent they are given. In addition, this report also examines how the BYOD approach is implemented and with what effects to learning situations.
\end{abstract}

Bock, Katharina. 2019. «Autorität von Medientechnik - Effekte sozio-materieller Arrangements in der Schule». MedienPädagogik, (Oktober), 82-100. https://doi.org/10.21240/mpaed/00/2019.10.17.X. 


\section{Einführung}

Zahlreiche Publikationen widmen sich der Bedeutung und Wirkung von Räumen, Architektur und Dingen in der Bildungspraxis (vgl. u.a. Breidenstein 2004; Rittelmeyer 2004; Rabenstein und Wienike 2012; Nohl und Wulf 2013). Das Zusammenspiel sozialer Aktivitäten mit materiellen Entitäten und mögliche, damit einhergehende machtvolle Effekte finden bisher allerdings wenig Beachtung (vgl. Alkemeyer und RiegerLadich 2008).

Soziale bzw. menschliche Aktivitäten können als mit der materiellen Umwelt verflochten, als mit ihr verwoben, begriffen werden (vgl. Schatzki 2016, 69), denn diese Umwelt

«begleitet uns nicht bloß, sie zieht uns an und stößt uns ab, sie fordert uns auf und ignoriert uns, sie versorgt uns mit Ideen, Motiven und Bedürfnissen, sie blockiert uns durch offensichtliche Barrieren und lenkt uns hintergründig, ohne dass wir dies durchschauen» (Hackl 2015, 132).

Ein hierfür weit gefasster Materialitätsbegriff schliesst sowohl Organismen (z.B. Natur, Tiere) ein, als auch Zeichen, Schrift und graphische Systeme (z.B. Typographie, Schrift, Landkarten), Materialien (z.B. Farbe, Pigmente, Stoffe), Räume und Artefakte (z.B. Bauwerke, Computer, Werkzeuge, Apparaturen); ebenso physikalische Phänomene (z.B. Licht, Klänge, Geräusche) und Substanzen (z.B. Wasser, Luft) (vgl. Kalthoff et al. 2016, 11f.). Mit dieser Perspektive geht es also um die Frage danach, wie das Materielle auf menschliches Handeln wirkt; es ermöglicht, beschränkt, de-/stabilisiert usw. (vgl. Kalthoff et al. 2016, 21).

In diesem Zusammenhang werden auch Hierarchie- und Machtverhältnisse relevant. Diese lassen sich mit dem Konzept der pädagogischen Autorität greifen. Dabei handelt es sich um eine spezifische Form sozialer Macht, die zwanglos akzeptiert, geachtet und anerkannt wird (vgl. Helsper et al. 2007, 52; Paris 2009, 38). Pädagogische Autorität hat zentrale Werte, grosse Kompetenzen, Stärke und Souveränität zu (re-)präsentieren, besitzt Vorbildcharakter und macht Eindruck. Zudem erbringt sie Leistungen, die gleichzeitig die Grundlage ihres Prestiges darstellen und ihre Anerkennung sichern sollen (vgl. Helsper et al. 2007, 45; Paris 2009, 39). Wer Autorität besitzt, hat die Macht anzuordnen, zu sanktionieren, zu urteilen, Gratifikationen zu gewähren etc., was bedeutet durch Autorität werden Ungleichheit und Hierarchie konstituiert (vgl. Helsper et al. 2007, 46). Entgegen der gängigen Auffassung, dass Autorität unbedingt (und zwar meist durch Lehrpersonen) verkörpert sein muss (vgl. Paris 2009, 39; Helsper et al. 2007, 45), sind in der hier eingenommenen Perspektive Hierarchie- und Machtverhältnisse nicht ausschliesslich an menschliche Körper und deren Aktivitäten gebunden, sondern können auch durch Formen des Materiellen hervorgebracht werden. 
Mein Beitrag zeichnet den Materialisierungsprozess pädagogischer Autorität i.S. hierarchisch-machtvoller Strukturen nach und zeigt, auf welchem Weg technische Geräte und Infrastrukturen Macht erlangen. Dafür wird vielfältiges ethnografisches Datenmaterial (s.u.) zu einem kapitelweise aufeinander aufbauenden, sich schrittweise verdichtenden ethnografischen Bericht entwickelt, analytisch reflektiert und interpretiert (vgl. u.a. Bock 2019, Absätze 8-10).

Wie sich zeigt, wird dieser Materialisierungsprozess an der untersuchten Schule massgeblich unterstützt durch einen >Bring Your Own Deviceく (BYOD)-Ansatz. Dieser Ansatz gilt als Versuch, die private, medial gute Ausstattung von Kindern und Jugendlichen (vgl. MPFS 2018 und jünger) auch im schulischen Kontext zu nutzen (vgl. Schiefner-Rohs et al. 2013, 14). Von bestimmten Schüler/innen wird demnach erwartet, sich selbst finanzierte, portable, internetfähige Geräte anzuschaffen und diese, insbesondere für bestimmte Unterrichtsfächer immer dabei zu haben. Der ethnografische Bericht arbeitet heraus, welche Bedeutung die Schule bzw. deren (didaktische) Leitung medienbezogenen Lehr-/Lernprozessen und dem BYOD-Ansatz zuschreibt, welche konzeptuellen und technischen Voraussetzungen daran geknüpft und inwieweit diese gegeben sind. Zudem wird herausgearbeitet, wie der BYOD-Ansatz realisiert wird und welche Effekte die Art der Umsetzung insbesondere für die sbetroffenen ‘ Schüler/innen hat. Diese, wie sich zeigt, finden sich in wirkmächtigen, von der Schule (bzw. deren didaktischer Leitung) induzierten, sozio-materiellen Arrangements wieder, die ihre Arbeits- und Partizipationsmöglichkeiten einzuschränken und die erstrebten pädagogischen Vorteile des Einsatzes von (privater) Medientechnik vorzuenthalten vermögen.

\section{Untersuchungsdesign}

Der vorliegende Beitrag basiert auf Datenmaterial, das ich im Rahmen des Projekts «Materialität von Geschlecht und pädagogischer Autorität - Interferenzen von Körpern und Dingen in Bildungsinstitutionen» an einer Integrierten Gesamtschule gewonnen habe. Hierbei handelt es sich um ein vom niedersächsischen MWK von 2017 bis 2019 im Rahmen des Programms «Geschlecht, Macht, Wissen» gefördertes Verbundprojekt zwischen der Leuphana Universität Lüneburg, der Technischen Universität Braunschweig sowie der Universität Hildesheim.

An der untersuchten Schule werden Klassen ganztags, vom Primar- bis in den Sekundarstufenbereich II unterrichtet. Die Schule verfolgt einen antiautoritären Ansatz (man duzt sich) und ein inklusives, barrierefreies Konzept. 


\section{Theoretischer Hintergrund}

Im Fokus dieses Forschungsprojekts stehen sozio-materielle Arrangements ${ }^{1}$ und u.a. die Frage, wie sich im Zusammenwirken von menschlichen Aktivitäten und räumlichdinglichen Gegebenheiten pädagogische Autorität materialisiert. Der Prozess des Zusammenwirkens von menschlichen Körpern, Dingen und Räumen lässt sich mit Bezug auf Butler als «Materialisierung» verstehen:

«Was ich [...] vorschlagen möchte, ist eine Rückkehr zum Begriff der Materie, jedoch nicht als Ort oder Oberfläche vorgestellt, sondern als ein Prozeß der Materialisierung, der im Laufe der Zeit stabil wird, so daß sich die Wirkung von Begrenzung, Festigkeit und Oberfläche herstellt, den wir Materie nennen.» (Butler 1997, 32)

Materie, i.S.v. Welt und Bedeutungsbildungen in ihr, kann so als im Werden aufgefasst werden. Damit lässt sich auch pädagogische Autorität als etwas Prozesshaftes begreifen, das im Laufe der Zeit stabilisiert und verfestigt wird.

Das Verhältnis von Materialität und Sozialität lässt sich einerseits als ein situiertes und Sinn konstituierendes Handeln mit Formen des Materiellen, andererseits als wirkmächtige Rahmung sozialen Handelns durch diese Formen verstehen (vgl. Kalthoff et al. 2016, 12). Mit dieser Forschungsperspektive lässt sich die materielle Dimension jedweder Praxis in all ihren Details berücksichtigen:

«Materialität ist dann nicht bloße Eigenschaft klar umrissener Entitäten, sondern als Sozio-Materialität immer schon verschränkt mit allem, was wir tun und beobachten. [...] Die Graduierung von Materialität und Sozialität hat ihre Spiegelung in theoretischen Ansätzen, die mal die Macht der Dinge, der Technik oder des Materials, mal die Herrschaft des Individuums über seine Werkzeuge betonen. So heben manche Autor[inn]en insbesondere die symbolische Qualität materieller Objekte hervor, während andere das Handeln-Können als ein Zusammenwirken von materiellen Objekten und sie verwendenden Akteur[inn]en beschreiben. Sind materielle Entitäten also in den Augen der Einen einzig und allein Werkzeuge im Dienste des Menschen, so sind sie bei den Anderen gleichwertige Akteur[inn]e[n], die ein Geschehen ermöglichen und Zeit und Raum - wenn auch unsichtbar - miteinander verbinden; sehen die Einen ihre Relevanz insbesondere in der Symbolisierung einer spezifischen sozialen Ordnung, so die Anderen in der Präjudizierung der sozialen Wirklichkeit». (Kalthoff et al. 2016, 14f.)

1 «Tatsächlich ereignet sich das soziale Leben [...] stets als Teil eines Gewebes aus Praktiken und Arrangements: Praktiken werden ausgeübt inmitten von und bestimmend für, zugleich aber auch in Abhängigkeit von und beeinflusst durch materielle Arrangements». (Schatzki 2016, 70) 


\section{Forschungsstrategie}

Das Projekt verfolgte eine ethnografische Forschungsstrategie (Breidenstein et al. 2015; de Boer und Reh 2012), die verschiedene Verfahren der Datengewinnung und ihrer Analyse kombinierte. Dazu gehörten Erhebungen aus teilnehmenden Beobachtungen in unterschiedlichen Unterrichts- und Pausensituationen, aus ethnografischen Gesprächen ${ }^{2}$ und offenen Leitfadeninterviews ${ }^{3}$ mit Lehrkräften und Schüler/ innen. Diese wurden ergänzt durch Recherchen sowie das Erstellen und Sammeln zahlreicher schulkultureller Artefakte (z.B. Lagepläne, Raumskizzen) und Dokumente (z.B. interne Dokumente wie das schulische Medienkonzept, das Schulentwicklungskonzept, Anmeldeunterlagen, Informationsschreiben). Der Offenheit (i.S. einer partiellen Befreiung von Methodenzwängen) in der Phase der Datengewinnung entsprach auch ein offener und explorativer Umgang mit den Methoden der Datenanalyse (vgl. Breidenstein et al. 2015, 111). Mit Hilfe der Methode der Ethnographischen Semantik (Spradley 1979; 1980; Maeder und Brosziewski 1997) wurde das Material zunächst grob erschlossen ${ }^{4}$ und systematisiert. Dem schloss sich das Interpretieren ausgewählter Szenenbeschreibungen ${ }^{5}$ an, die eine besonders prägnante Verschränkung von Materialität und Autorität aufwiesen. Hierüber liessen sich nicht nur verschiedene Modi der De-/Stabilisierung von Autorität (z.B. Ausdehnung, Delegation, Umkehrung) identifizieren, sondern auch Materialisierungsmodi (d.h. Materialisierung von Autorität z.B. über gesprochene Sprache, über Mimik und Körperbewegungen, über sinnliche Wahrnehmungen, über räumliche Positionierungen, über Bildlichkeit). Diese Modi öffneten den Blick für das Prozesshafte der Hervorbringung von Autorität. Vor diesem Hintergrund wurde das Datenmaterial dann final zunächst offen kodiert und anschliessend kategorisiert, wodurch sich bereits vorhandene Modi präzisieren, aber auch zusätzliche Modi identifizieren liessen.

2 Ethnografische Gespräche dienten dazu, das vor allem durch teilnehmende Beobachtungen, aber auch aus Recherchen, Dokumenten und Artefakten erworbene Wissen über das Untersuchungsfeld zeitnah, kontext- und situationsgebunden zu hinterfragen, zu reflektieren und etwaige Verständnisfragen klären zu können. Im Unterschied zu klassischen (halb-)standardisierten Interviews betonen ethnografische Gespräche den informellen Charakter der Konversationen. Solche, in Umgangssprache geführten Unterhaltungen ohne Hierarchien und Rollenzuweisungen sind erfahrungsgemäss essentiell für das Verständnis dessen, was vor Ort geschieht (vgl. Dellwing und Prus 2012, 114ff.).

3 Offene, leitfadengestützte Interviews wurden vor allem gegen Ende des Feldaufenthalts geführt. Auch diese Befragungen zeichneten sich durch einen eher informellen Charakter aus; zudem waren sie deutlich ausführlicher und von deutlicher zeitlicher Ausdehnung. Im Zentrum dieser Befragungen standen Themen wie der jeweilige berufliche Werdegang, Fragen zum Umgang mit und Einsatz von Medien(-inhalten, -anwendungen, -geräten) für Schul- und Unterrichtszwecke sowie Fragen zu schulbezogenen Zahlen und Fakten. Zudem ging es um das Erfassen von Medienbegriffs- und Autoritätsannahmen. Die Interviews dienten ausserdem dem Reflektieren bestimmter, z.T. gemeinsam erlebter, Situationen sowie der Klärung von Widersprüchen und Ambivalenzen. Darüber hinaus ermöglichte das vorhandene Vertrauensverhältnis auch kritische Nachfragen.

4 Nach Personen, Orten, Handlungen und Dingen.

5 Dabei handelt es sich um Beobachtungsprotokolle, die mit zusätzlichem Datenmaterial angereichert und so zu elaborierten ethnografischen Beschreibungen ausgebaut wurden. 
Sozio-technische Arrangements in medienbezogenen Lehr-/Lernprozessen

\section{Konzeptuelle Voraussetzungen}

Mit ihrem internen Medienkonzept verfolgt die untersuchte Schule bzw. deren (didaktische) Leitung das Ziel der «Anbahnung von Medienkompetenz» ${ }^{6}$. Der dort verwendete Medienbegriff bezieht sich sowohl auf «die klassischen Medien Buch, Zeitung, Plakat, Film und Fernsehen» als auch auf «die auf digitaler computertechnischer Basis arbeitenden vernetzten Multimediatechnologien». Unter Bezugnahme auf Baacke (1997), Schorb (2005) und Vollbrecht (2001) definiert die Schule bzw. deren (didaktische) Leitung «Medienkompetenz« für sich als »die Fähigkeit und Fertigkeit, Medien im privaten und beruflichen Kontext bedienen zu können, [...] mediale Phänomene wie soziale Netzwerke etc. kritisch zu reflektieren und unsere Medienlandschaft aktiv mitzugestalten». Hieraus leitet sie zwei Handlungsfelder ab (Angebote auswählen und nutzen; Beiträge gestalten und verbreiten), die sie um drei Inhalts- und Reflexionsbereiche ergänzt (Sprache der Medien verstehen und bewerten; Wirkung von Medien erkennen und aufarbeiten; Bedingungen der Produktion und Verbreitung von Medien in ihren gesellschaftlichen Kontexten durchschauen und beurteilen). Vergleicht man die Medienkompetenzauffassung der Schule mit den Anforderungen an «Kompetenzen in der digitalen Welt», wie sie die KMK (2016) formuliert, zeigen sich grosse umfangsmässige und inhaltliche Unterschiede: Im Vergleich zur Schule formuliert die KMK sechs Kompetenzbereiche (Suchen, Verarbeiten und Aufbewahren; Kommunizieren und Kooperieren; Produzieren und Präsentieren; Schützen und sicher Agieren; Problemlösen und Handeln; Analysieren und Reflektieren), die jeweils detailreich ausdifferenziert werden. Die grössten inhaltlichen Leerstellen aufseiten der Schule bestehen bezüglich Kommunikations-, Interaktions-und Kooperationskompetenzen, hinsichtlich rechtlicher Kompetenzen (Wissen über Urheber-, Nutzungs- und Persönlichkeitsrechte), hinsichtlich Kompetenzen zur Datenspeicherung und dem Treffen notwendiger Schutzmassnahmen (Wissen über Datensicherheit, Jugend-, Verbraucherschutz, Schutz von Gesundheit, Natur und Umwelt) (vgl. KMK 2016, 16-19).

Wie aus dem internen Medienkonzept und dem internen Schulentwicklungsprogramm hervorgeht, spielt medienbezogenes Lernen bzw. die Nutzung geeigneter Medienangebote im Unterricht für die untersuchte Schule eine wichtige Rolle. Zur Umsetzung dessen wurden fächer- und themenbezogene «Medienbausteine» entwickelt, die «obligatorisch» zum Einsatz kommen (sollen). Diese Bausteine werden allerdings nur für den Primar- und Sekundarstufenbereich I formuliert. Auf mein Nachfragen

6 Die Anführungszeichen markieren die Verwendung von Feldsprache. Ich halte die Leser/innen für fachkundig genug, zwischen Feldbegriffen/Feldzitaten auf der einen und herkömmlichen Literaturzitaten auf der anderen Seite zu differenzieren. 
zeigt sich die Schulleitungsebene diesbezüglich überrascht; das Fehlen von Medienbausteinen für die Sekundarstufe II kann sie nicht begründen. Die vorhandenen Bausteine beinhalten den Einsatz verschiedener Medienträger (CDs und DVDs), den Umgang mit und das Erstellen von unterschiedlichen Medieninhalten (u.a. Poster, Broschüren, Fotokollagen, Open Office-Präsentationen, Hörspiele oder kurze Videos) sowie Internetrecherchen. Hinweise auf bestimmte digitale Lernsoftware oder Lernmaterialien aus dem Internet fehlen - obwohl derartige Programme etwa im Mathematikunterricht zum Einsatz gebracht werden. Dies legt nahe, dass die Schule solche Lehr-/Lernangebote als nicht (für alle Fächer gleichermassen) geeignet erachtet.

Eine besondere Rolle spielt der Einsatz privater Medientechnik im Unterricht. Einen BYOD-Ansatz - ohne den Begriff jedoch explizit zu benutzen - verfolgt die Schule in der Sekundarstufe I seit 2016. Die Einrichtung solcher Klassen erfolgt dort bislang optional und ist abhängig von der Zustimmung und Finanzierungsbereitschaft aller Eltern einer Klasse - die Schule erwägt allerdings, die selbstverständliche Nutzung privater Geräte perspektivisch von nahezu allen Schüler/innen zu erwarten. Im Sekundarstufenbereich II wird der Ansatz seit bereits ungefähr zehn Jahren verfolgt; hier ist er für alle Schüler/innen verpflichtend. Dem internen Medienkonzept nach, verfolgen diese (BYOD-)Notebook-/Tablet-Klassen das Anliegen, die Geräte als «multimediale Lehr- und Lernwerkzeuge» zu nutzen. Sie sollen die Schüler/innen motivieren, sie «audiovisuell quasi ımehrspurig» informieren und innen «durch aktuelle, lebensnahe, multimediale Unterrichtsmaterialien» ein »pädagogisches Mehr« zu realisieren helfen - näher ausgeführt und/oder mit Kompetenzbereichen verknüpft wird dieses Mehr allerdings nicht. Mittels Strategien zur «zielführenden Informationsbeschaffung und -verarbeitung» sollen die Schüler/innen darüber hinaus vielfältige Informationsmöglichkeiten nutzen, Informationen visualisieren, präsentieren und verbreiten lernen. Hier zeigen sich die erwähnten Leerstellen in der Medienkompetenzauffassung der Schule: Rechtsfragen, Sicherheits- und Schutzmassnahmen spielen offenbar keine bedeutsame Rolle. Hinzu kommt, dass für den Ansatz selbst keine detaillierten jahrgangs-, fächer- und themenspezifischen medienpädagogischen Strategien, Werkzeuge und/oder Ressourcen vorliegen. Laut Schulentwicklungsprogramm befinden sich diese zum Zeitpunkt der Untersuchung noch in der Entwicklung $^{7}$, was weitere Leerstellen aufzeigt. Wie aus dem erwähnten Schulentwicklungskonzept, aus Gesprächen mit Vertreter/innen der Schulleitung sowie mit der für das aktuelle Tablet-Projekt verantwortlichen Lehrperson hervorgeht, steht auch eine (Zwischen-)Evaluation der BYOD-Projekte aus. Zu beobachten sei aber, so erzählt mir diese Lehrperson, dass die Schüler/innen zunehmend an grosser Selbstsicherheit im Umgang mit den Geräten, deren Funktionen und Strukturen gewinnen und lernen

7 Rudimentäre bzw. noch in der Erarbeitung befindliche Medienkonzepte sind an (deutschen) Schulen kein Einzelfall. Die Gründe dafür liegen vor allem in den für die konzeptuelle Arbeit fehlenden Ressourcen sowie darin, dass die Arbeit am Medienkonzept im Vergleich zu anderen Aufgaben nicht als vordringlich erachtet wird (vgl. Schaumburg et al. 2007, 42). 
würden, sich in der Medienwelt zu bewegen und damit kritisch umzugehen. ${ }^{8}$ Basis der (Weiter-)Arbeit mit privater Medientechnik im Unterricht bilden demnach Beobachtungen und daraus abgeleitete Vermutungen über ein vages pädagogisches Mehr. Dies legt nicht nur einen Mangel an der notwendigen Ernsthaftigkeit und Systematik bei der Umsetzung des BYOD-Ansatzes nahe, sondern lässt auch Kompetenzdefizite aufseiten der Lehrkräfte vermuten.

Potenziale des BYOD-Ansatzes ergeben sich nur dann, wenn der Einsatz der privaten Mediengeräte im Unterricht «zielgerichtet und bewusst» erfolgt (Schiefner-Rohs et al. 2013, 15). Hierfür allerdings fehlen die konzeptuellen Voraussetzungen, denn Grundlage der medienpädagogischen und -didaktischen Arbeit der Schule ist ein unterkomplexes, lückenhaftes Medien(aneignungs)konzept, das konkreten Lehr-/Lernwerkzeugen für die betreffenden Klassen entbehrt und den Anforderungen an ein Lernen mit und über digitale Medien im 21. Jhd. nicht gerecht wird. Lehrpersonen insbesondere in BYOD-Klassen sind offenbar weitgehend auf sich selbst zurückgeworfen, weil es an der notwendigen Grundlage und Unterstützung fehlt. Zudem verhindert das Ausbleiben von (zumindest Zwischen-)Evaluationen die Weiterentwicklung und Optimierung medienbezogener Lehr-/Lernprozesse, und der suboptimale Status quo läuft Gefahr, im Laufe der Zeit stabilisiert und verfestigt zu werden. Insgesamt macht das bisher präsentierte Datenmaterial grossen Nachholbedarf deutlich. Um den Auftrag «Bildung in der digitalen Welt» (KMK 2016) verantwortungsvoll und hinreichend erfüllen zu können, müssen deutlich mehr Ressourcen bemüht und muss inhaltlichkonzeptionell sowie praktisch-methodisch sehr viel stärker investiert werden.

\section{Nutzung (medien-)technischer Ausstattung und Infrastruktur}

Dem Einsatz von Medientechnik im Unterricht schreibt die untersuchte Schule grosse Bedeutung zu. Dies wird u.a. deutlich an mit (herkömmlichen) Whiteboards und Beamern ausgestatteten Räumen, an der zunehmenden Ausstattung bestimmter Räume mit Smart- und VISU-Boards sowie an der Einrichtung von BYOD-Notebook-/TabletKlassen. ${ }^{9}$

8 Eine Leistungssteigerung würde übrigens nicht erwartet. Diese Vermutung wird durch Studien bestätigt (vgl. u.a. Häuptle und Reinmann 2006; Schaumburg und Issing 2002; Schaumburg et al. 2007): Zwar werden «Potenziale in den Bereichen Schüler[innen]aktivität, Selbstständigkeit und Motivation» vermutet, aber «der Mehrwert des Unterrichts mit Notebooks» sei vermutlich «nicht primär in der Verbesserung von Fachleistungen zu suchen [...] oder zumindest nur dann, wenn hier deutlich mehr Ressourcen als bisher investiert werden, um Konzepte für den fachdidaktisch sinnvollen Einsatz zu entwickeln und die Lehrkräfte auch entsprechend fortzubilden» (Schaumburg et al. 2007, 125).

9 Ausserdem arbeitet die Schule mit «iServ», einer Verwaltungs- und Organisations-, Kommunikations- und Lernplattform. Wie mir Schüler/innen und Lehrpersonen gleichermassen berichten, beschränkt sich die Nutzung von iServ für Unterrichts- und Lernzwecke vor allem auf das Verfügbarmachen von und Zugreifen auf Dateien wie Arbeitsblätter, Vertretungs-, Termin- und Klausurpläne. Audiovisuelle Inhalte oder digital gespeicherte Tafelbilder würden eher selten und nicht in allen Fächern verfügbar gemacht. Das Mailingprogramm komme zwar zum Einsatz, präferiert aber würden Gruppenchatmöglichkeiten externer Messenger-Dienste. Die Befragten beschreiben iServ als fehler-, störanfällig und z.T. dysfunktional, was eine nachvollziehbare Erklärung für die eher basalen Nutzungsweisen der Plattform liefert. 
Wie eingangs erwähnt, müssen Schüler/innen der Oberstufe ihre privaten Geräte, insbesondere für den Mathematikunterricht, immer dabeihaben. Ausserdem sind sie zum Herunterladen und Installieren der kostenfreien Mathematik-Software «GeoGebra» verpflichtet. In der Mathematikstunde einer elften Klasse beobachte ich folgende Situation: Die Klasse hat von Paula, der Lehrerin, gerade ein Arbeitsblatt mit Aufgaben ausgehändigt bekommen, und diese Aufgaben sollen die Schüler/innen nun mit Hilfe von GeoGebra lösen. Während dieser Arbeitsphase ist plötzlich ein leiser, elektronischer Ton zu hören. Zeitgleich erscheint auf dem Laptop-Bildschirm des direkt vor mir platzierten Schülers Konstantin eine kleine Anzeige, die ihm meldet, dass der «Akkustand» seines Geräts «niedrig» ist, verbunden mit der Aufforderung, das Gerät an eine Stromquelle anzuschliessen. Dieser Aufforderung kommt Konstantin nicht nach. Stattdessen fährt er sein Gerät herunter und verstaut es anschliessend in seinem Rucksack. Er tut dies, so vermute ich, weil es keine von seinem Sitzplatz aus erreichbare Stromquelle für inn gibt. Paula reagiert darauf nicht. Konstantin wird am heutigen Unterrichtsgeschehen erst wieder partizipieren, als es eine Aufgabe händisch auf Papier zu lösen gilt. Wie ich anschliessend in der Pause überprüfen werde, verfügt dieser Raum über genau zwei Steckdosen, und zwar jeweils eine etwa mittig unterhalb der beiden frontal zur Klasse ausgerichteten Whiteboards (vgl. Abb. 1). Diese beiden Stromquellen, so schlussfolgere ich, sind wohl am besten vom Lehrer/innentisch aus zu erreichen; möglicherweise ausserdem noch von einigen günstig platzierten Schüler/innen in der ersten Reihe. Allerdings, so nehme ich an, würde letztere Nutzungsweise wahrscheinlich eine grosse Stolpergefahr provozieren (- nicht unproblematisch in einer als «barrierefrei» geltenden Schule). Auch Schaumburg et al. $(2007,56)$ berichten von solchen Stromversorgungsproblemen: die Akkulaufzeiten reichen für die Länge des Schultags nicht aus und in den Unterrichtsräumen sind nicht genügend Steckdosen vorhanden bzw. befinden sich nicht in der Nähe der Schüler/innentische. Dadurch werden die Gerätenutzungs- und die Partizipationsmöglichkeiten von Schüler/innen in BYOD-Klassen stark eingeschränkt. 


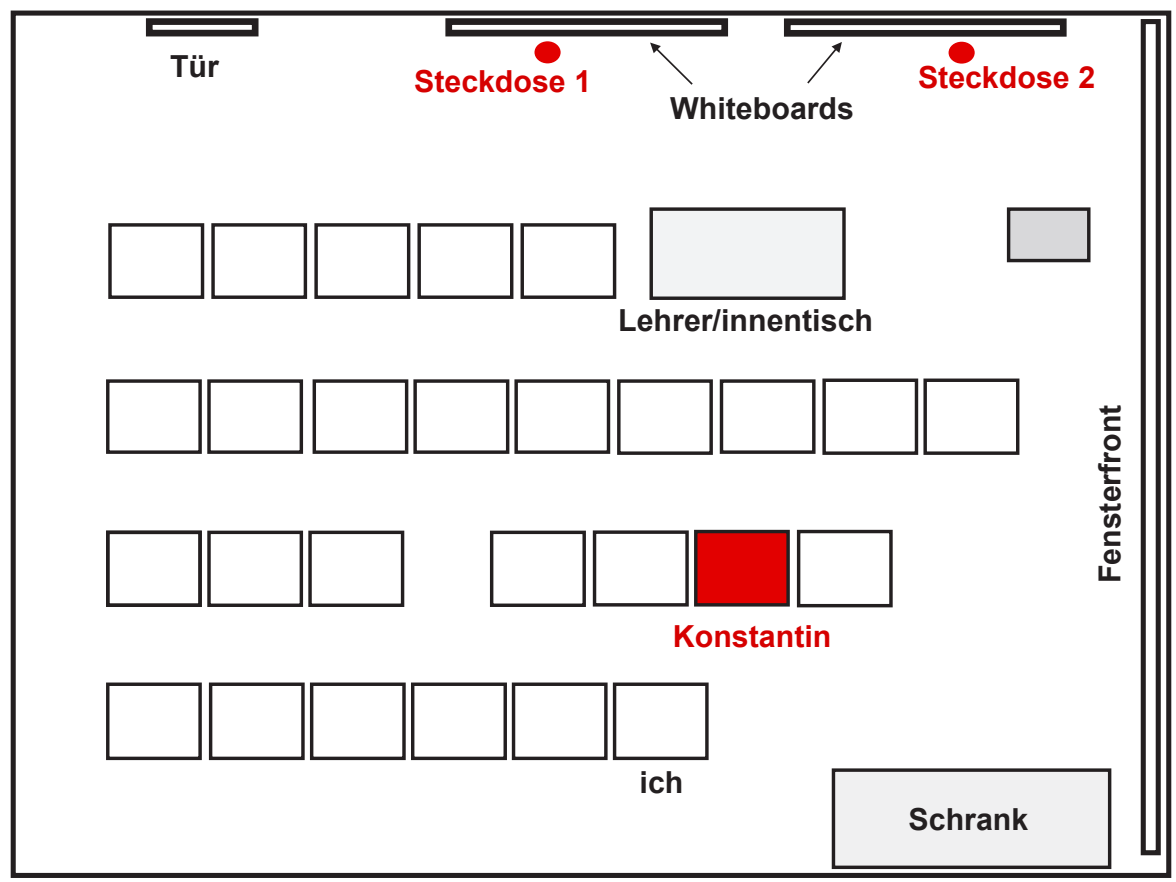

Abb. 1.: Raumskizze Mathematikunterricht (eigene Darstellung).

Zu einem späteren Zeitpunkt im Feld wohne ich dem Politikunterricht dieser Klasse bei. Hier beobachte ich u.a. folgende Situation: Julius, der Politiklehrer, betritt den Raum und steuert auf den Lehrer/innentisch zu. Dort legt er eine grosse Tasche ab, in der sich ein portabler schwarzer Beamer befindet - der in diesem Raum an der Decke angebrachte Beamer ist zu diesem Zeitpunkt schon eine Weile defekt. (Das Zurückgreifen auf portable Geräte ist üblich, da nicht alle mit Whiteboards ausgestatteten Räume über einen eigenen Beamer verfügen.) Den Beamer platziert Julius auf einem kleinen, nebenstehenden Tisch. Aus einer weiteren Tasche holt er ein silberfarbenes Apple-Notebook. Dieses Notebook, wird mir Julius später erzählen, ist sein privates Gerät. Es sei einfacher, sein eigenes Gerät zu nutzen, sagt er. Scheinbar gibt es nicht für jeden Fachbereich ein solches Gerät, und auch nicht in ausreichender Zahl. Julius ist ausserdem noch Musiklehrer, und auch für diesen Unterricht bereite er sehr viel an seinem privaten Notebook vor, arrangiere kleine Sachen und visualisiere sie, und zwar von daheim aus.

Nun leitet Julius über zum eigentlichen Unterricht. Er würde heute Einblick in ein neues Thema geben. Das werde per Video passieren. "Kurze Frage: Hier ist 'ne Videoanlage, aber keine Musikanlage? Warum ist drüben eine und hier nicht? Hat jemand 'ne tragbare Box dabei?», fragt Julius. Die Schülerin Rike meldet sich und erklärt, sie habe eine Box, aber nur mit Bluetooth. Mit dieser Box geht sie nach vorne zu Julius, um auszuprobieren, ob der Einsatz ihrer Box möglich ist - ist er nicht, wie 
beide umgehend feststellen. Daraufhin gibt der Schüler Ergün zu verstehen, er habe ebenfalls eine Box, mit Kabel. Julius und Ergün unternehmen daraufhin einen weiteren Versuch, das Notebook mit einem Lautsprecher zu verbinden. Nachdem sie eine Weile an den Geräten herumgefummelt haben, schaut Julius plötzlich hoch, richtet seinen Blick auf den Schüler Daniel und fragt diesen, ob er wisse, warum das nicht funktioniere. «Dann ist das Kabel kaputt», lautet Daniels Diagnose. «Gut, dann müssen wir das vom Laptop aus machen», sagt Julius und macht sich auf den Weg zur Tür. «Dann müssen wir jetzt mal ganz leise sein», lässt er die Klasse wissen, während er die Tür schliesst. Bei dem vorzuführenden Video handelt es sich um einen kurzen ZDF heute-Magazinbeitrag zum Thema «Mikroplastik in der Arktis». Diesen Beitrag hat Julius bei YouTube aufgerufen und spielt inn nun ab. (Glücklicherweise besteht gerade eine Internetverbindung - das ist nicht immer der Fall.) Dabei projiziert er das Video nun auf das rechte von zwei verfügbaren Whiteboards - und zwar leicht schief, ohne weitere Vergrösserung und ziemlich weit nach rechts zum Fenster hingerückt. Die potentiell zur Verfügung stehende Gesamtprojektionsfläche nutzt er so gar nicht voll aus. Der aufgrund fehlender Lautsprecher nun nur vom Notebook ausgehende Ton ist bloss mit grosser Konzentration zu verstehen. Hinzu kommt auch noch eine schlechte Sicht, denn der projizierte Medieninhalt ist zu klein und ziemlich hell (vgl. Abb. 2 und 3).

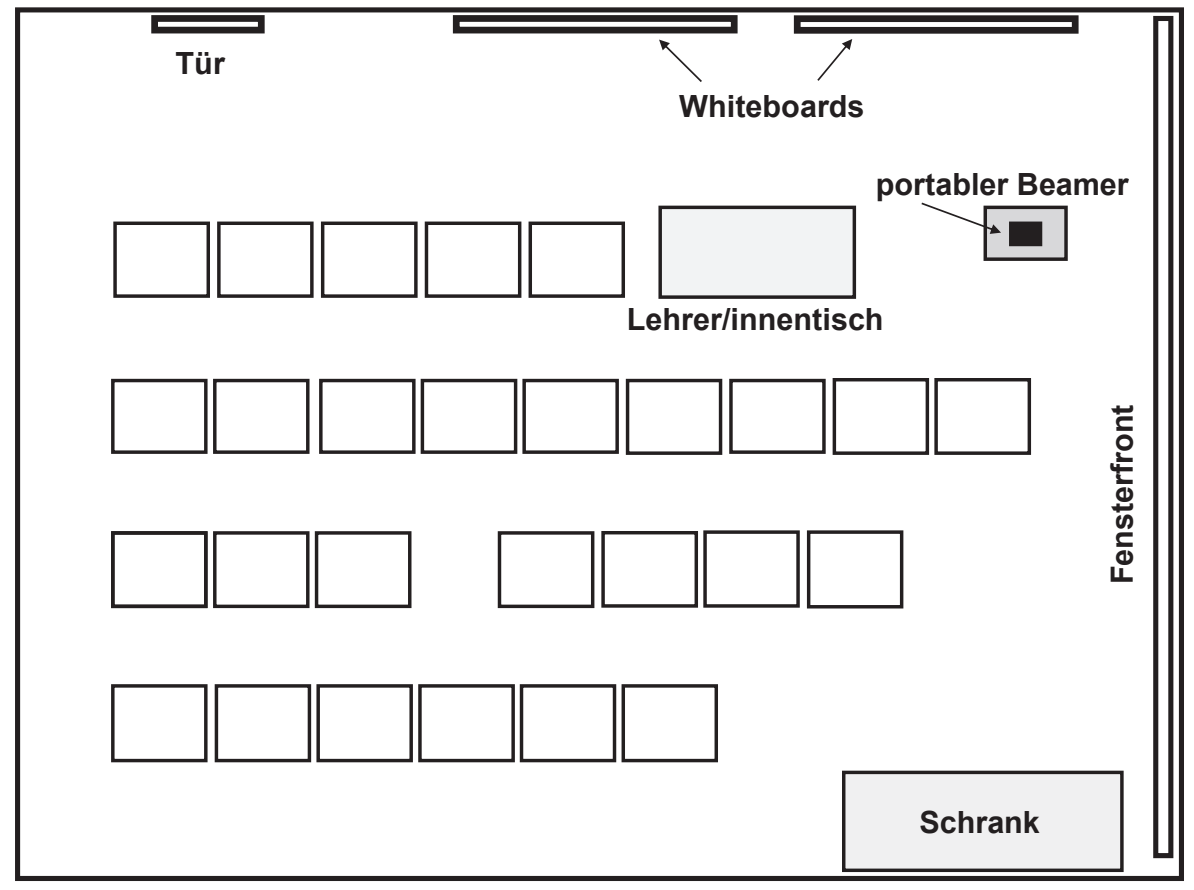

Abb. 2.: Raumskizze Politikunterricht (eigene Darstellung). 


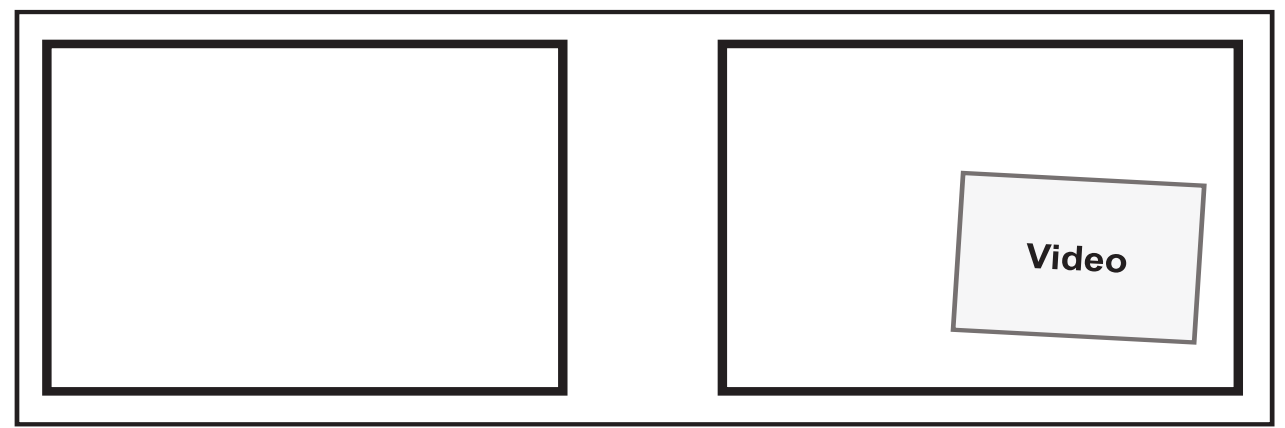

Abb. 3.: Skizze Whiteboard-Nutzung (eigene Darstellung).

"Weil es zu Beginn doch noch etwas unruhig war», wie Julius findet, spielt er das Video anschliessend noch ein zweites Mal ab. Und abermals haben wir uns auf Bild und Ton in besonderer Weise zu konzentrieren. Dies alles kostet viel Zeit, wie auch Maeder $(2015,172)$ feststellt: «What ... surprises systematic observers is how much time is needed in the classroom to get the ICT running in the desired way: ICT use in classroom imposes a never-ending series of situated repair work (SRW) to all users». Die fehlenden Lautsprecher (als ein Mangel in der medientechnischen Infrastruktur der Schule) und Julius' Versäumnis, eine optimale Sicht auf den Medieninhalt zu gewährleisten, stellen ein sozio-technisches Arrangement dar, das sich negativ auf die Rezeptions- bzw. Lernsituation auswirkt und den Schülerinnen und Schülern ein zusätzliches Mehr an Konzentration auf Ton und Bild abverlangt.

Nach dem Unterricht unterhalte ich mich noch kurz mit Julius. Während er Notebook und Beamer wieder in den Taschen verstaut, kommentiere ich: «Mensch, musst du das hier alles jedes Mal anschleppen?». «Hör mir auf», antwortet Julius kopfschüttelnd, "die Medienausstattung hier an der Schule ist 'ne Katastrophe. Ich war vorher woanders und da war alles vom Allerfeinsten. Und dann kam ich hierher und - naja». Hier sei ständig irgendwas kaputt oder würde fehlen. Man könne sich auf nichts verlassen und müsse immer irgendwie improvisieren. Diesbezüglich sehe er an dieser Schule hier noch viele Entwicklungsmöglichkeiten. In einem späteren Gespräch wird Julius mir erzählen: Anders als an der vorherigen Schule, an der er war, müsse er sich hier mit der Technik an jeden Raum neu anpassen. Es gebe zwar in der Oberstufe bereits sehr viele Smartboards, aber dann würden teilweise die Kabel fehlen, das Handling sei anders - mal gebe es 'ne Tastatur, dann wieder nicht; mal gebe es einen Adapter, dann wieder nicht. Das koste alles viel Zeit. Im Musikbereich zum Beispiel gebe es gar keine Smartboards, und er frage sich warum. Gerade in Musik seien Smartboards besonders wichtig, weil man multimedial einfach viel machen könne, sagt er. Dort gebe es schlichte Whiteboards. Auf die könne man draufschreiben und fertig. Selbst Dranprojizieren sei schwierig, denn für den Musikbereich gebe es nur einen portablen Beamer - für sieben Musiklehrer/innen. Eben diesen einen Beamer hatte er für die beschriebene Politikstunde übrigens von dort mitgebracht, sagt er. 
Als ich den Raum nach dem Politikunterricht schliesslich verlasse, grüsst mich die Referendarin Vera aus dem mir bereits bekannten Unterrichtsraum gegenüber und wir kommen kurz ins Gespräch. Währenddessen macht sich Vera daran, die beiden äusseren beschriebenen Flügel eines Smartboards abzuwischen und kommentiert mit einigem Frust: Das müsse man immer alles gleich abwischen, denn das brenne sich richtig ein. Das mache aber immer keiner und dann sehe es halt richtig scheisse aus. Einen Lappen gebe es dafür natürlich auch nicht. Dieser hier sei ihr eigener, sagt sie auf das Mikrofasertuch in ihrer Hand deutend; den habe sie immer dabei. Dann fährt Vera fort: Sie habe vor einem halben Jahr hier angefangen und da habe man ihr bereits gesagt, das Ding sei kaputt. Seitdem sei auch nichts passiert. Sie rechne auch nicht damit, dass es noch repariert werde. Und selbst wenn, dann würden irgendwelche Kabel fehlen - immer sei irgendwas. Das Ding sei auch echt blöd: man könne nur die beiden äusseren Flügel beschreiben - die Mitte bekäme man nicht mehr abgewischt, ausserdem sei sie nicht mal magnetisch.

Die Beobachtungen und Schilderungen zeigen etliche Mängel in der z.T. basalen technischen Infrastruktur der Schule auf. Ein störanfälliges Netzwerk, defekte Beamer und Smartboards, eine unzuverlässige Internetverbindung, Stromversorgungsprobleme, fehlende Lautsprecher und Kabelverbindungen etc. determinieren die Unterrichts- und Lernbedingungen der betroffenen Schüler/innen und Lehrpersonen, schränken Mediennutzungsweisen und Partizipationsmöglichkeiten ein. Mit dieser Problemgemengelage umzugehen, Mängel zu kompensieren und sich immer wieder zu arrangieren, geht nicht nur zulasten wertvoller Unterrichtszeit. Vielmehr fordert eine für alle Beteiligten didaktisch halbwegs Erfolg bringende Nutzung der (privaten) Medientechnik im Unterricht den betroffenen Schüler/innen und Lehrpersonen ein erhebliches Mehr an Weitblick, Engagement, Konzentration, Verantwortungsbewusstsein und Improvisationstalent ab. Im Zusammenspiel von Sozialität und Materialität, so lässt sich schlussfolgern, wird die von der Schule angestrebte Umsetzung von Lehr-/Lernprozessen mit Multimediatechnologien deutlich konterkariert.

\section{Effekte sozio-technischer Arrangements}

Wie die vorangegangenen Ausführungen bereits andeuten, materialisieren sich im Verwoben-sein von Sozialität und Materialität zugleich Ungleichheits- und Machtverhältnisse. (Pädagogische) Autorität wird hier längst nicht mehr ausschliesslich an menschliche Körper gebunden, sondern hat sich auf materielle Formen ausgedehnt: Der Materialisierungsprozess von Autorität nimmt seinen Ausgang mit der Bedeutungszuschreibung, die medienbezogenes Lernen und der Einsatz von Medientechnik durch die Schule bzw. deren (didaktische) Leitung erfahren. Vor allem durch den verfolgten BYOD-Ansatz werden hierbei technische Geräte zu bedeutsamen Artefakten erklärt und erlangen selbst Autorität. Der Prozess setzt sich fort in Versäumnissen 
der Schule, die für eine sinnvolle und zielgerichtete Umsetzung medienbezogener Lehr-/Lernprozesse notwendigen Bedingungen zu schaffen. Dies betrifft die erwähnten konzeptuellen Defizite, die mangelhafte technische Ausstattung und Infrastruktur sowie das Ausbleiben von Evaluationen der schulischen Arbeit mit Medien(-technik). Auf diesem Weg werden sowohl technische Artefakte als auch Infrastrukturen symbolisch mit Macht (vgl. Bourdieu 2005, 82) ausgestattet und so in die Lage versetzt, die Unterrichtsbedingungen von Lehrpersonen und Schüler/innen zu determinieren.

Zusätzlich gestärkt wird diese Wirkmacht durch die Art der Umsetzung des verfolgten BYOD-Ansatzes. Machtvolle Effekte ergeben sich hier zum einen aus den Angaben zur Anschaffung der privaten Geräte: Dem Eltern-Informationsbogen zufolge sei auf eine technisch ausreichende Ausstattung (einen Prozessor mit mindestens 1 $\mathrm{GHz}$, mind. 2 GB RAM und mind. 32 GB Festplattenspeicher) zu achten. Anschaffen könne man sowohl Geräte mit einem Betriebssystem von Microsoft, Apple als auch Linux. Ausser Acht gelassen wird dabei, dass unterschiedliche Geräte aufgrund ihres unterschiedlichen Marken-Prestiges sowie unterschiedlicher, daran geknüpfter Preisniveaus geeignet sind, soziale Ungleichheit unter den Schüler/innen zu (re-)produzieren (vgl. Stager 2011; Schiefner-Rohs et al. 2013, 14) - ein Effekt, der deutlich im Widerspruch zum integrativen Konzept der Schule steht. Dem Informationsbogen zufolge sei ausserdem auf ausreichend lange Akku-Laufzeiten zu achten - genaue Richtwerte werden allerdings nicht genannt. Auch Hinweise auf besonders leistungsfähige Geräte gibt es nicht. Als ich Paula, die Mathematiklehrerin frage, ob es öfter vorkomme, dass die Akkus der Schüler/innen raussteigen und wie mit diesem Problem umgegangen werde, erklärt sie mir nüchtern: Die Laptops lägen in der Eigenverantwortung der Schüler/innen. Diese seien verpflichtet sicherzustellen, dass die Akkus geladen und die Geräte einsatzbereit sind. Würden die Schüler/innen feststellen, dass es ein Problem mit dem Akku gibt, dann müssten sie eben ein Ladekabel und notfalls auch eine Verlängerungsschnur dabeihaben. Dies zeigt: Die Gesamtverantwortung für die im Unterricht eingesetzten Privatgeräte wird an die Schüler/innen delegiert. Diese haben sich die erforderlichen Geräte nicht nur selbstfinanziert anzuschaffen, sondern auch die Verantwortung für deren Wartung, für technische Probleme oder etwaige Reparaturen zu tragen..$^{10} \mathrm{Um}$ ihre Partizipation im Unterricht zu gewährleisten, haben die Schüler/innen sicherzustellen, dass die Geräte jederzeit einsatzbereit sind. Dies allerdings erfordert ggf. nicht nur privates Zusatzequipment, sondern übrigens auch Schutz- und Zusatzsoftware (kostenpflichtige Anti-Viren-, Textverarbeitungs-, Präsentationssoftware etc.) - Informationen, die der ElternInformationsbogen ebenfalls nicht enthält. Aber nicht nur für Schüler/innen ergibt sich ein mit der Art der BYOD-Umsetzung einhergehender Mehraufwand. Aus arbeitsökonomischen Gründen und mangels ausreichender technischer Ausstattung der

10 Das Nicht-Vorhandensein technischer Administrator/innen gilt als bedeutendes Hemmnis der Integration digitaler Medien in die Schule (vgl. Schiefner-Rohs et al. 2013, 11). 
Schule werden auch Lehrpersonen implizit dazu veranlasst, ihre privaten Geräte für den Unterricht einzusetzen und darüber hinaus selbsttätig offenbar sogar fehlendes oder defektes Zubehör (Beamer, Lautsprecher, Kabel, Mikrofasertücher) zu kompensieren. Vor diesem Hintergrund scheint der BYOD-Ansatz vor allem als "pragmatische Lösung des technisch geprägten Ausstattungsdilemmas" (Schiefner-Rohs et al. 2013, 1) herzuhalten. Folgerichtig müsste der Ansatz hier eher lauten: 'BYOD - AEEYMN (Bring Your Own Device - And Anything Else You Might Need).

Der BYOD-Ansatz, so lässt sich schlussfolgern, kommt hier weitgehend unvorbereitet, unsystematisch, unreflektiert und deshalb höchst folgenreich zur Anwendung. Die entstehenden sozio-technischen Arrangements sind insofern wirkmächtig, als dass sie einen unverhältnismässigen Mehraufwand produzieren. Zudem vermögen sie die Arbeitsbedingungen von Lehrpersonen zu erschweren, die Unterrichtspartizipation von Schüler/innen einzuschränken und soziale Ungleichheit unter ihnen zu (re-)produzieren. Auf diese Weise bleiben den Betroffenen die erstrebten pädagogischen und didaktischen Vorteile des Einsatzes von (privater) Medientechnik im Unterricht nicht nur vorenthalten - mehr noch werden technische Artefakte und Infrastrukturen hier zu Medien der Vermittlung einer Schulkultur, die den Anforderungen der Digitalisierung im 21. Jhd. (noch) nicht gewachsen ist.

Indem die Betroffenen sich den Schwierigkeiten, Einschränkungen und Mängeln anpassen und den expliziten Vorgaben wie impliziten >Regeln ‘ der Schule Folge leisten - und dies bereits offenbar seit vielen Jahren - scheinen sie die für sie suboptimalen Rahmenbedingungen zu akzeptieren. Auf diesem Weg wird symbolische Macht bzw. Gewalt ${ }^{11}$ (vgl. Bourdieu 2005, 82; 2007, 265) ausgeübt, d.h.: Die betroffenen Schüler/innen und Lehrpersonen zeigen Formen der Beugung und Unterwerfung, die sie nicht als solche wahrnehmen, weil auf einer symbolisch-sinnhaften Ebene des Selbstverständlichen und Alltäglichen operiert wird (vgl. Schmidt und Woltersdorff 2008: 8). Aus den Versäumnissen der Schule leiten sich aufseiten der Betroffenen also psycho-physische Zwänge ab; sie verorten sich selbsttätig in von Machtkämpfen geprägten sozialen Räumen und akzeptieren ihre relationalen Platzierungen (vgl. Alkemeyer und Rieger-Ladich 2008, 114). Und indem sich die Betroffenen den von der Schule induzierten ssozio-technischen Macht-Arrangements، immer wieder unterordnen, tragen sie dazu bei, die bestehenden Verhältnisse auf Dauer zu stellen.

11 «Für das Feld der symbolischen Kämpfe unterscheidet Bourdieu nicht immer eindeutig zwischen den Begriffen Macht und Gewalt». (Audehm 2001, 104) 


\section{Schlussfolgerungen}

Pädagogische Autoritäten haben zentrale Werte, grosse Kompetenzen, Stärke, Souveränität und Sicherheit zu (re-)präsentieren; sie haben Vorbildcharakter zu besitzen und Eindruck zu machen. Sie haben Leistungen zu erbringen, die gleichzeitig die Grundlage ihres Prestiges darstellen und ihre Anerkennung sichern sollen (vgl. Helsper et al. 2007, 45; Paris 2009, 39). Bei der Implementierung von Innovationen und neuen Technologien haben allen voran die (didaktischen) Leitungen der Schulen die Verantwortung zu tragen; ihnen komme diesbezüglich eine Schlüsselrolle zu (vgl. Gerick et al. 2016, 60). Die zahlreichen Defizite und Versäumnisse, die Delegation der eigenen Verantwortung und das fehlende Bewusstsein für die Effekte sozio-materieller Dimensionen stellen die (Amts-, Funktions- und Organisations-)Autorität (vgl. Helsper et al. 2007, 50-52) der untersuchten Schule in Zweifel.

Obwohl die Schule die Aneignung von Medienkompetenz zum Bildungsziel erklärt und medienbezogenem Lernen sowie dem Einsatz von Medientechnik im Unterricht besondere Wertigkeit zugeschreibt, spiegelt sich diese Bedeutsamkeit auf Unterrichtsebene nicht wider. Dies zeigt, dass die Integration neuer Technologien in Unterrichtsentwicklungsprozesse Schulen (noch immer) vor grosse Herausforderungen stellt (vgl. Gerick et al. 2016, 62; 84). Um dem Auftrag »Bildung in der digitalen Welt« (KMK 2016) gerecht zu werden, haben Schulen wesentliche Gelingensbedingungen zu schaffen. Diese müssen zunächst die Entwicklung geeigneter und durchdachter medienpädagogischer Konzepte umfassen. Hierfür allerdings ist es notwendig,

«das klassische Verständnis der Medienkompetenz aufzubrechen und dieses in Verbindung mit informatischen sowie interdisziplinären sozialwissenschaftlichen Kompetenzen zur Digitalen Bildung im Sinne einer Befähigung zur kritischen Informations- und Medienkompetenz in der Informations-, Medien- und Wissensgesellschaft zu erweitern» (Dengel 2018, 24)..$^{12}$

Darüber hinaus gilt es, konkrete Massnahmen zur Herstellung funktionierender technischer Infrastrukturen zu entwickeln. ${ }^{13}$ Dazu wiederum gehören die Ausstattung mit einem leistungsfähigen Internetzugang, die Sicherstellung einer ausreichenden Stromversorgung in BYOD-Klassenräumen und der Aufbau verzahnter externer und interner Wartungs- und Supportstrukturen. Zudem sollten konkrete Empfehlungen bestimmter, und zwar robuster Hardware, ausgesprochen werden - im Idealfall speziell für die Nutzung in der Schule konstruierte Geräte, die den Belastungen des Schulalltags dauerhafter standhalten als herkömmliche Geräte (vgl. Schaumburg et al. 2007, 126f.).

12 Dabei sieht Dengel $(2018,24)$ insbesondere auch die Hochschulen in der Verantwortung: Bevor die Digitale Bildung Einzug in die Lehrpläne finde, müsse sie zunächst an den Hochschulen vermittelt werden, um qualifizierte Lehrpersonen hervorzubringen, welche diese Inhalte auch unterrichten können.

13 Mehr Unterstützung hierbei leistet künftig der «Digitalpakt Schule», der den flächendeckenden, modernen digitalen Infrastrukturen in Deutschlands Schulen den Weg bereiten soll (vgl. BMBF 2019). 


\section{Literatur}

Alkemeyer, Thomas, und Markus Rieger-Ladich. 2008. «Symbolische Gewalt im pädagogischen Feld: Überlegungen zu einer Forschungsheuristik». In Symbolische Gewalt. Herrschaftsanalyse nach Pierre Bourdieu, hrsg. v. Robert Schmidt und Volker Woltersdorff, 103-124. Konstanz: UVK.

Audehm, Kathrin. 2001. «Die Macht der Sprache. Performative Magie bei Pierre Bourdieu». In Grundlagen des Performativen. Eine Einführung in die Zusammenhänge von Sprache, Macht und Handeln, hrsg. v. Christoph Wulf, Michael Göhlich und Jörg Zirfas, 101-128. Weinheim, München: Juventa.

Baacke, Dieter. 1997. Medienpädagogik. Tübingen: Niemeyer.

Breidenstein, Georg. 2004. «KlassenRäume - eine Analyse räumlicher Bedingungen und Effekte des Schülerhandelns». In Zeitschrift für qualitative Bildungs-, Beratungs- und Sozialforschung 5: 1, 87-107.

Breidenstein, Georg, Stefan Hirschauer, Herbert Kalthoff, und Boris Nieswand. 2015 [2013]. Ethnografie. Die Praxis der Feldforschung, 2., überarb. Auflage. Konstanz, München: UVK.

Bock, Katharina. 2019. «Ethnografisches Protokollieren - Erkenntnisabsichten und sprachlichstilistische Gestaltungsprinzipien» [56 Absätze]. In Forum Qualitative Sozialforschung / Forum: Qualitative Social Research, 20(1), Art. 6. http://www.qualitative-research.net/index. $\mathrm{php/fqs/article/view/2933.}$

Bourdieu, Pierre. 2005 [1992]. »Die verborgenen Mechanismen der Macht enthüllen«. In Die verborgenen Mechanismen der Macht. Schriften zu Politik und Kultur 1, 81-86. Hamburg: VSA Verlag.

Bourdieu, Pierre. 2007. «Politisches Feld und symbolische Macht. Gespräch mit Effi Böhlke». In Bourdieu und die Linke. Politik - Ökonomie - Kultur, hrsg. v. Effi Böhlke und Rainer Rilling, 263-270. Berlin: Dietz.

BMBF - Bundesministerium für Bildung und Forschung. 2019. «Bund und Länder über DigitalPakt Schule einig. 5,5 Milliarden für die digitalen Infrastrukturen der Schulen - Beitrag zur digitalen Bildung in Deutschland», Pressemitteilung 018/2019 vom 15.03.2019. https:// www.bmbf.de/de/bund-und-laender-ueber-digitalpakt-schule-einig-8141.html.

Butler, Judith. 1997. Körper von Gewicht. Die diskursiven Grenzen des Geschlechts. Frankfurt am Main: Suhrkamp.

De Boer, Heike, und Sabine Reh. 2012. Beobachtung in der Schule - Beobachten lernen. Wiesbaden: Springer VS.

Dellwing, Michael, und Robert Prus. 2012. Einführung in die interaktionistische Ethnografie. Soziologie im Außendienst. Wiesbaden: Springer VS.

Dengel, Andreas. 2018. «Digitale Bildung: ein interdisziplinäres Verständnis zwischen Medienpädagogik und Informatik». In MedienPädagogik, Themenheft 33, Medienpädagogik und Didaktik der Informatik. Eine Momentaufnahme disziplinärer Bezüge und schulpraktischer Entwicklungen, hrsg. v. Torsten Brinda, Ira Diethelm, Sven Kommer und Klaus Rummler. https://doi.org/10.21240/mpaed/33/2018.10.30.X. 
Gerick, Julia, Birgit Eickelmann, Kerstin Drossel, und Ramona Lorenz 2016. «Perspektiven von Schulleitungen auf neue Technologien in Schule und Unterricht». In ICILS 2013. Vertiefende Analysen zu computer- und informationsbezogenen Kompetenzen von Jugendlichen, hrsg. v. Birgit Eickelmann, Julia Gerick, Kerstin Drossel und Wilfried Bros, 60-92. Münster, New York: Waxmann.

Hackl, Bernd. 2015. «Zimmer mit Aussicht. Räumlichkeiten als Medium von Bildungsprozessen». In Bildungspraxis. Körper, Räume, Objekte, hrsg. v. Thomas Alkemeyer, Herbert Kalthoff und Markus Rieger-Ladich, 131-158. Weilerswist: Velbrück.

Helsper, Werner, Heiner Ullrich, Bernhard Stelmaszyk, Davina Höblich, Gunther Graßhoff, und Dana Jung. 2007. Autorität und Schule. Die empirische Rekonstruktion der KlassenlehrerSchüler-Beziehung an Waldorfschulen, 1. Auflage. Wiesbaden: Verlag für Sozialwissenschaften.

iServ. o.J. Schulserver. https://iserv.eu/portal/philosophy/.

Kalthoff, Herbert, Markus Rieger-Ladich, und Thomas Alkemeyer. 2015. «Bildungspraxis - eine Einleitung». In Bildungspraxis. Körper, Räume, Objekte, hrsg. v. Thomas Alkemeyer, Herbert Kalthoff und Markus Rieger-Ladich, 9-33. Weilerswist: Velbrück.

Kalthoff, Herbert, Torsten Cress, und Tobias Röhl. 2016. «Einleitung: Materialität in Kultur und Gesellschaft». In Materialität. Herausforderungen für die Sozial- und Kulturwissenschaften, hrsg. v. Herbert Kalthoff, Torsten Cress und Tobias Röhl, 11-41. Paderborn: Wilhelm Fink Verlag.

KMK - Kultusministerkonferenz. 2016. »Bildung in der digitalen Welt Strategie der Kultusministerkonferenz i.d.F.v. 07.12.2017«. https://www.kmk.org/fileadmin/Dateien/veroeffentlichungen_beschluesse/2018/Strategie_Bildung_in_der_digitalen_Welt_idF._ vom_07.12.2017.pdf.

Maeder, Christoph. 2015. «ICT in Classrooms. The Practical Side of a Technical Order». In MultiPluriTrans in Educational Ethnography. Approaching the Multimodality, Plurality and Translocality of Educational Realities, hrsg. v. Sabina Bollig, Michael-Sebastian Honig, Sascha Neumann und Claudia Seele, 163-174. Bielefeld: Transcript.

Maeder, Christoph und Achim Brosziewski. 1997. «Ethnographische Semantik: Ein Weg zum Verstehen von Zugehörigkeit». In Sozialwissenschaftliche Hermeneutik. Eine Einführung, hrsg. v. Ronald Hitzler und Anne Honer, 335-362. Opladen: Leske + Budrich.

MPFS - Medienpädagogischer Forschungsverbund Südwest. 2018. «JIM-Studie 2018. Jugend Information, Medien. Basisuntersuchung zum Medienumgang Jugendlicher 12- bis 19-Jähriger». https://www.mpfs.de/fileadmin/files/Studien/JIM/2018/Studie/JIM_2018_Gesamt. pdf.

Nohl, Arnd-Michael, und Christoph Wulf. 2013. «Die Materialität pädagogischer Prozesse zwischen Mensch und Ding». In Zeitschrift für Erziehungswissenschaft, Sonderheft 25: Mensch und Ding. Die Materialität pädagogischer Prozesse, hrsg. v. Arnd-Michael Nohl und Christoph Wulf, 1-13. Wiesbaden: Springer VS.

Paris, Rainer. 2009. »Die Autoritätsbalance des Lehrers«. In Autorität, hrsg. v. Alfred Schäfer und Christiane Thompson, 37-63. Paderborn u.a.: Schöningh. 
Rabenstein, Kerstin, und Johanna Wienike. 2012. «Der Blick auf die Dinge des Lernens. Überlegungen zur Beobachtung der materiellen Dimension pädagogischer Praktiken». In Beobachtung in der Schule - Beobachten lernen, hrsg. v. Sabine Reh und Heike de Boer, 189-202. Wiesbaden: Springer VS.

Rittelmeyer, Christian. 2004. «Zur Rhetorik von Schulbauten. Über die schülergerechte Gestaltung des architektonischen Ausdrucks». In Die Deutsche Schule 96: 2, 201-208.

Schatzki, Theodore R. 2016. «Materialität und soziales Leben». In Materialität. Herausforderungen für die Sozial- und Kulturwissenschaften, hrsg. v. Herbert Kalthoff, Torsten Cress, und Tobias Röhl, 63-88. Paderborn: Wilhelm Fink Verlag.

Schaumburg, Heike, Doree Prasse, Karin Tschackert, und Sigrid Blömeke. 2007. „Lernen in Notebook-Klassen. Endbericht zur Evaluation des Projekts $>1000$ mal1000: Notebooks im Schulranzen«" https://www.researchgate.net/publication/238769876_Lernen_in_ Notebook-Klassen_Endbericht_zur_Evaluation_des_Projekts_1000mal1000_Notebook s_im_ Schulranzen/download.

Schiefner-Rohs, Mandy, Richard Heinen, und Michael Kerres. 2013. «Private Computer in der Schule: Zwischen schulischer Infrastruktur und Schulentwicklung». In MedienPädagogik 13: 2013, 1-20. https://doi.org/10.21240/mpaed/00/2013.04.30.X.

Schmidt, Robert, und Volker Woltersdorff. 2008. Symbolische Gewalt. Herrschaftsanalyse nach Pierre Bourdieu. Konstanz: UVK.

Schorb, Bernd. 2005. «Medienkompetenz». In Grundbegriffe Medienpädagogik, 4., vollständig neu konzipierte Auflage, hrsg. v. Jürgen Hüther und Bernd Schorb, 345-351. München: kopaed Verlags $\mathrm{GmbH}$.

Spradley, James. 1979. The ethnographic interview. New York, NY: Holt, Rinehart and Winston. Spradley, James. 1980. Participant observation. New York, NY: Holt, Rinehart and Winston.

Stager, Gary. 2011. «BYOD - Worst Idea of the 21st Century?». http://stager.tv/blog/?p=2397.

Vollbrecht, Ralf. 2001. Einführung in die Medienpädagogik. Weinheim, Basel: Beltz.

\section{Danksagung}

Ich danke den Gutachterinnen und Gutachtern für ihre konstruktiven Anregungen und Hinweise. 